\title{
Leveraging complex interactions between signaling pathways involved in liver development to robustly improve the maturity and yield of pluripotent stem cell-derived hepatocytes
}

Claudia Raggi ${ }^{1,5}$, Marie-Agnès M'Callum $^{1}$, Quang Toan Pham ${ }^{1}$, Perrine Gaub ${ }^{2,5}$, Silvia Selleri ${ }^{1}$, Nissan Baratang ${ }^{5}$, Chenicka Lyn Mangahas ${ }^{1}$, Gaël Cagnone ${ }^{2}$, Bruno Reversade ${ }^{3}$, Jean-Sébastien Joyal $^{2,4}$, Massimiliano Paganelli, ${ }^{1,2,4,6}$

${ }^{1}$ Liver Tissue Engineering and Cell Therapy Laboratory, ${ }^{2}$ CHU Sainte-Justine Research Center, Montreal, Canada; ${ }^{3}$ Institute of Molecular and Cell Biology and Institute of Medical Biology, A*STAR, Singapore; ${ }^{4}$ Department of Pediatrics, Université de Montréal, Montreal, Canada; ${ }^{5}$ Morphocell Technologies Inc., Montreal, Canada; ${ }^{6}$ Pediatric Hepatology, CHU Sainte-Justine, Montreal, Canada.

Correspondence: m.paganelli@umontreal.ca, @maxpaganelli 


\section{SUMMARY}

Pluripotent stem cell (PSC)-derived hepatocyte-like cells (HLC) have shown great potential as an alternative to primary human hepatocytes $(\mathrm{PHH})$ for in vitro modeling. Several differentiation protocols have been described to direct PSC towards the hepatic fate, although the resulting HLC have shown more a fetal than adult phenotype. Here, by leveraging recent knowledge of the signaling pathways involved in liver development, we describe a robust, scalable protocol that allows to consistently generate high-quality HLC from both ESC and iPSC. Such HLC are comparable to adult PHH in terms of key mature liver functions and proved suitable to assess drug hepatotoxicity, as a proof of concept of their potential as a physiologically representative alternative for in vitro modeling. 


\section{INTRODUCTION}

For decades, the study of human liver physiology and development, as well as drug discovery, have been limited by the lack of representative models. Animal studies and in vitro models based on tumor cell lines proved crucial to build our current knowledge but are inadequate to assess the specificities of the human liver. Primary human hepatocytes (PHH) suffer from major limitations that have hampered our ability to create proper in vitro human models and generate representative data. Although considered the "gold standard" by regulatory agencies, PHH do not allow a reliable prediction of a drug's metabolism and toxicity in human subjects, which results in a high failure rate at clinical phases and is partially responsible of the high costs and long times of drug development.

Stem cell-derived hepatocyte-like cells $(\mathrm{HLC})$ have shown great potential as an alternative to $\mathrm{PHH}$. Pluripotent stem cells (PSC), such as embryonic stem cells (ESC) and induced pluripotent stem cells (iPSC), are a potentially unlimited source from which to produce HLC. ${ }^{1}$ Various differentiation protocols inspired by liver organogenesis have been described for both ESC and iPSC. Empirical identification of best culture conditions has historically played a central role in establishing such protocols. Our knowledge of liver development has greatly advanced over the last decade and we now have a reasonable understanding of the signaling pathways involved. ${ }^{2-}$ ${ }^{4}$ This helped to improve the quality of PSC-derived HLC. ${ }^{5-7}$ Nevertheless, obtained HLC are still closer to fetal than adult $\mathrm{PHH}^{8}$ Moreover, the quality of differentiation varies widely among HLC obtained from different PSC populations, and cell yield is often low with most of described protocols. ${ }^{9}$ These limitations constitute a significant barrier to the full implementation of HLC for in vitro modeling, drug testing and cell therapy applications. By leveraging most recent knowledge of signaling pathways involved in liver development and timely acting on Wnt/ $\beta$ catenin, oncostatin $\mathrm{M}(\mathrm{OSM})$ and TGF $\beta$ pathways during the later stages of differentiation, we developed a new robust protocol allowing for high-yield, consistent generation of high-quality bipotent liver progenitors and HLC from both ESC and iPSC. 


\section{RESULTS}

We used 6 different PSC populations (one ESC line and five iPSC lines) and differentiated them through consecutive stages following liver development (Fig. 1A,B).

\section{Hepatic specification}

After careful characterization of PSC (Supplementary Fig. S1), we recreated the process of gastrulation in vitro, passing by a primitive streak stage and then pushing Brachyury $(\mathrm{T})$-positive cells towards the mesendoderm through epithelial-mesenchymal transition by concurrently activating both Activin/Nodal signaling (with Activin A) and canonical Wnt/ $\beta$-catenin pathway (via the selective inhibition of GSK3 $\beta$ with CHIR99021). This led to the timed upregulation of EOMES and FOXA2 (24 hours) and SOX17 (48 hours; Fig. 2A). Stimulation of Activin/Nodal pathway for 3 additional days allowed the cells to acquire the gene and protein expression profile typical of the definitive endoderm (DE; Fig. 2B,C). The obtained DE population was highly homogenous, with a $>90 \%$ of FOXA2, SOX17 and CXCR4-positive cells (Fig. 2D,E). The addition of knock-out serum replacement (KOSR) at low concentration, which, as fibroblast grow factor (FGF) secreted by the cardiac mesoderm, induces hepatic specification through the MAPK signaling pathway, significantly reduced cell death without affecting the quality of the obtained DE (Supplementary Fig. S2A,B). No difference was seen in the results obtained from applying this protocol to ESC or iPSC populations (Supplementary Fig. S2C).

In the embryo, the DE is then exposed to BMP from septum transversum mesenchyme (STM) and FGF from the cardiac mesoderm (Fig. 1A). ${ }^{2,3}$ This drives the cells towards the formation of the posterior foregut. Inhibition of Wnt signaling pathway is needed to relieve the repression of HHEX, which is required for the commitment to the hepatic fate. ${ }^{10}$ TGF $\beta$ inhibition allows for upregulation of albumin $(A L B)$ and PROX1 genes, the latter playing an essential role in subsequent liver bud formation. ${ }^{11}$ After 5-day exposure to BMP4, low-dose FGF, A83-01 (TGF $\beta$ pathway inhibitor) and IWP2 (Wnt pathway inhibitor), the cells acquired a phenotype suggestive of the commitment of the ventral posterior foregut (VPFG) towards the hepatic fate 
(corresponding to mouse embryonic day E8.5), with the overexpression of HHEX, PROX1 (Fig. 2F), HNF4a, $\alpha$-fetoprotein (AFP) and ALB genes (Fig. 2G).

\section{Liver bud formation and expansion}

In humans, 9 days post-gastrulation, hepatic progenitor cells from the VPFG delaminate and invade the surrounding stroma, forming the liver bud (LB). Liver progenitors (hepatoblasts) enter hence into contact with the STM, which continue secreting BMP, and are in close proximity with sinusoidal endothelial cells secreting Wnt and hepatocyte growth factor (HGF; Fig. 1A). Supplementation of hepatoblasts with BMP4, FGF, HGF and CHIR99021, in the presence of insulin, for 5 days, triggered the re-establishment of cell-cell contacts and a deepest commitment towards the hepatic fate (Fig. 1B). At the end of this stage, such LB cells further increased the expression of transcription factors HNF4a and of PROX1, both required for their proliferation and differentiation (Fig. $2 \mathrm{H}$ ). ${ }^{11,12}$ ALB and AFP continued to be very highly expressed in LB cells. The expression of HNF1b, which at this stage controls cholangiocyte fate, was instead reduced, while the expression of SRY-box 9 (SOX9) was significantly increased in LB cells, which is consistent with the expression of these genes in bipotent hepatic progenitor cells (Fig. $2 \mathrm{H}$ ). ${ }^{13}$ Immunostaining showed that $79.1 \% \pm 13.4 \%$ of LB cells were AFP-positive, $80.4 \% \pm$ $10 \%$ were albumin-positive and $97.2 \% \pm 2.7 \%$ EpCAM-positive (Fig. 2I). The expression of cytokeratin 19 (CK19) was ubiquitous, although variable between cells and significant in only $36.3 \% \pm 28 \%$ of them. EpCAM staining at the cell surface confirmed the re-establishment of cell-cell contacts (Fig. 2J). The number of cells still expressing pluripotency markers was negligible at this stage (Fig. 2J). The staged activation of HHEX, HNF4a and PROX1 also led to the proliferation of hepatoblasts (Fig. $2 \mathrm{~K}$ ), in line with the behavior of the liver bud at this stage of development (day 24-48 of gestation, corresponding to E10-E14 in mouse). ${ }^{11}$ No difference between ESC and iPSC was noted (Supplementary Fig. S2D).

\section{Maturation into hepatocyte-like cells}

In the developing human embryo, starting at around gestational day 57 (E13.5 in mouse), the close contact between hepatoblasts and hepatic stellate cells (secreting HGF and FGF) and 
hematopoietic cells (producing OSM) drives their maturation into hepatocytes (Fig. 1A). Mature liver functions are then progressively acquired, with the liver expressing a fully mature phenotype only several weeks after birth. We exposed LB cells to OSM and dexamethasone in addition to the stimulation of BMP, FGF, HGF and Wnt signaling pathways and inhibition of TGF $\beta$ pathway, for 5 days, to induce maturation to HLC (Fig. 1B). Subsequently, we maintained only OSM and dexamethasone for another 5 days and then concluded the differentiation processes with further 5 days without OSM, in order to mimic the decrease in the stimulus from hematopoietic cells that happens in the later weeks of gestation. ${ }^{14}$ At the end of the differentiation process, we obtained a homogeneous monolayer of mono- and binucleated polygonal epithelial cells (Fig. 3A). Such PSC-derived HLC showed an important increase in the expression of $A L B$ ( $>1000$-fold change) and AFP ( $>10$-fold change) compared to LB hepatoblasts (Fig. 3B). The expression of mature liver-specific genes ALB, ASGR1 and TAT in HLC was comparable to $\mathrm{PHH}$ (Fig. $3 \mathrm{C}$ ), with HNF4a and AFP being more highly expressed. The differentiation protocol proved to be robust, with negligible inter-population and interoperator variability (32 differentiations of 6 PSC populations, 3 different operators; Supplementary Fig. S2E). No difference between ESC and iPSC was noted (Supplementary Fig. S2F). Albumin and AFP expression was very homogeneous in HLC (Fig. 3D). Interestingly, CK19 was expressed by $44.6 \% \pm 22.4 \%$ of the cells, most of which were also albumin positive. HLC effectively performed mature liver functions such as cytochrome P450 3A4 (Cyp3A4) activity, albumin secretion and urea production and resulted comparable to metabolism-qualified adult PHH (Fig. 3E).

As a proof of concept, we used such HLC to asses hepatotoxicity of two common drugs with a known dose-dependent effect on hepatocytes (Fig. 3F,G). Mitochondrial dysfunction has emerged as an important mechanism and indicator of drug-induced hepatotoxicity. ${ }^{15}$ By measuring their mitochondrial respiration (oxygen consumption rate, OCR), we showed that HLC allow to representatively assess, with very little variability, the toxic effect of acetaminophen and amiodarone on hepatocytes' metabolism.

When compared to previously published protocols for which a transcriptome was available ${ }^{7,16-}$ 18 and to a differentiation protocol not acting on the $W n t / \beta$-catenin and TGF $\beta$ signaling 
pathways beyond the definitive endoderm stage (Supplementary Fig. S3), our new protocol resulted in significantly more mature HLC (Fig. 4 and Supplementary Fig. S4). HLC obtained with our protocol significantly overexpressed key liver-specific genes compared to other previously described HLC and resulted in expression profiles more similar to PHH (Fig. 4A-C). A three-fold increase was found for those encoding albumin and enzymes involved in xenobiotic metabolism, iron homeostasis, and lipids and bile acid metabolism (Supplementary Fig. S4B,C). Analysis of Gene Ontology terms confirmed the overexpression of superclusters of genes that are essential for the development, structural integrity and function of the human liver (Supplementary Fig. S4D). The advantage of the timed regulation of Wnt/ $\beta$-catenin and TGF $\beta$ signaling pathways was even more evident when measuring mature liver functions (Fig. 4E). Moreover, our new protocol allowed to obtain significantly more HLC (Fig. 4F) and resulted in an upregulation of Hippo/YAP and Notch signaling pathways, which is suggestive of the regenerative/replicative state of the cells (Supplementary Fig. S4D) and well explains the better yield. $^{19}$

Overall, our new differentiation protocol allowed to consistently generate more mature HLC from both ESC and iPSC compared to previously described approaches, with good yield and using reagents that have Good Manufacturing Practices (GMP)-compliant versions available. Designed for robustness and ease of clinical translation, the protocol has been licensed to a regenerative medicine company, validated by 3 more independent operators and successfully tech-transferred to an independent contract development and manufacturing organization (CDMO) for follow-on scale-up and optimization.

\section{DISCUSSION}

Over the years, the differentiation protocols were progressively refined to generate more mature HLC from either ESC or iPSC by acting on very diverse signaling pathways. Nevertheless, despite the heterogeneity of growth factors, small molecules, culture media and coating compounds used, full maturity of adult hepatocytes has never been achieved in vitro, and a 
significant variability in the performance of many protocols across various pluripotent stem cell lines was often observed. ${ }^{8,9}$ Here we described a new protocol that, through the timed activation and inhibition of key signaling pathways playing a role along liver development, allows to reproducibly generate high-quality HLC from PSC. This protocol generates highly homogeneous populations at each stage of hepatogenesis, with no significant variability when applied to various IPSC and ESC populations. The obtained HLC were more mature than cells generated with previously described, widely used protocols not leveraging the timed and complex interaction between signaling pathways. Wnt/ $\beta$-catenin signaling plays a crucial role in orchestrating liver development. ${ }^{2-4}$ Although the role of this pathway during the later stages of development has been well shown in rodents, the need for its subsequent inhibition and activation in synergy with BMP, FGF, OSM and TGF $\beta$ signaling modulation has not been previously exploited to generate HLC from PSC. Our HLC expressed most of liver-specific genes responsible for the organ's synthetic and metabolic activity and performed significantly better at key mature liver functions than previously described protocols not simultaneously acting on such signaling pathways. Only hepatocytes obtained from fibroblasts by transdifferentiation through forced expression of specific hepatocyte transcription factors (hiHeps) showed a gene expression profile more similar to $\mathrm{PHH}$, although they clustered further than our HLC when only key liver-specific genes were considered. ${ }^{17}$ Most importantly, our HLC showed Cyp3A4 activity and albumin and urea production capabilities comparable to adult $\mathrm{PHH}$, and proved suitable for in vitro drug testing. Although metabolism-qualified cryopreserved PHH are known to lose functionality compared to freshly isolated $\mathrm{PHH}$, they are still considered the industry "gold standard" to assess candidate new drugs, despite the high inter-donor variability and high costs. $^{20}$

It is widespread experience that most differentiation protocols have an overall low yield, with significant cell death, which increases the costs of differentiation and limits scalability. The activation of $\mathrm{Wnt} / \beta$-catenin pathway was shown to have an important role on hepatocyte proliferation and might contribute to the good yield of our protocol. ${ }^{21}$ Such a better yield compared to protocols not relying as much on the modulation of TGF $\beta$ and Wnt pathways can also be explained by the upregulation of Hippo/YAP signaling shown above. Besides promoting 
cell proliferation, the activation of this pathway reduces apoptosis, and seem to play a key role in liver regeneration. ${ }^{19,22}$ Sustained Wnt activation during maturation of hepatoblasts into HLC and timed supplementation of OSM to mimic the physiological role of sinusoidal and hematopoietic cells residing in the liver during fetal life allowed for better maturation of our HLC compared to the ESC-derived cells previously described by Touboul et al. ${ }^{7}$ Avoidance of NOTCH inhibition resulted in higher SOX9 expression, but had no impact on mature liver function, while it further supported cell proliferation and reduced the cost of differentiation. Moreover, we avoided the use of serum and used reagents that are easily transitioned to GMPcompliant versions. This will reduce times and costs of GMP implementation and further eases the protocol's translation for future potential therapeutic applications. Successfully techtransferred to a cell therapy company and a CDMO, the protocol confirmed its robustness, although optimization will be needed to allow large scale production required for meaningful in vitro drug testing and cell therapy applications.

Overall, we described here a new differentiation protocol to consistently obtain high-quality, homogeneous and functional HLC with a high yield from both human ESC and iPSC. With low variability between starting PSC lines, this protocol might prove useful for liver disease modeling. Moreover, the protocol design and yield suggest good potential for scalability, which makes it promising for in vitro drug testing and development. Once their potential to replace mature liver functions in vivo proven, such HLC might also be considered for cell therapy applications.

\section{EXPERIMENTAL PROCEDURES}

A detailed description of all experimental procedures is provided as Supplementary material.

\section{PSC differentiation into hepatocyte-like cells (HLCS)}

PSCs grown in Essential 8 Flex medium (see Supplementary material for details on PSC generation, characterization and maintenance) were dissociated by TrypLE (Life Technologies) to single cells and seeded on human recombinant laminin 521 (BioLamina)-coated plates in 
Essential 8 Flex medium at a density of $7 \times 10^{4}$ cells $/ \mathrm{cm}^{2}$. Differentiation was started (day 0) when the cells reached $70 \%$ confluence by changing the medium to RPMI-B27 minus insulin (Life Technologies) supplemented with 1\% KOSR (Life Technologies). For the first 2 days, the cells were exposed to $100 \mathrm{ng} / \mathrm{ml}$ Activin A (R\&D Systems) and $3 \mu \mathrm{M}$ CHIR-99021 (Stem Cell Technologies), and then for the 3 following days to $100 \mathrm{ng} / \mathrm{ml}$ Activin A alone. Subsequently, RPMI-B27 (minus insulin) medium was supplemented with $20 \mathrm{ng} / \mathrm{ml} \mathrm{BMP4} \mathrm{(Peprotech),} \mathrm{5ng/ml}$ bFGF (Peprotech), $4 \mu \mathrm{M}$ IWP-2 (Tocris), and $1 \mu \mathrm{M}$ A83-01 (Tocris) for 5 days, with daily medium change. At day 10, the medium was changed to RPMI-B27 (Life Technologies), supplemented with 2\% KOSR, 20 ng/ml BMP4, 5 ng/ml bFGF, 20 ng/ml HGF (Peprotech) and $3 \mu$ M CHIR-99021 for 5 days, with daily medium change. At day 16, the medium was changed to HBM/HCM (minus EGF) medium (Lonza), supplemented with 1\% KOSR, $20 \mathrm{ng} / \mathrm{ml} \mathrm{HGF,} 20 \mathrm{ng} / \mathrm{ml} \mathrm{BMP4,} 5$ $\mathrm{ng} / \mathrm{ml}$ bFGF, $3 \mu \mathrm{M}$ CHIR-99021, $10 \mu \mathrm{M}$ dexamethasone (Sigma), and $20 \mathrm{ng} / \mathrm{ml}$ OSM (R\&D System) for 5 days, with daily medium change. From day 20, for 5 days, HBM/HCM medium was supplemented with $1 \%$ KOSR, $10 \mu \mathrm{M}$ dexamethasone and $20 \mathrm{ng} / \mathrm{ml}$ OSM, changing the medium every other day. From day 25 , the cells were maintained in HBM/HCM $1 \%$ KOSR medium supplemented with $10 \mu \mathrm{M}$ dexamethasone, with every other day medium change. During all the differentiation process, the cells were kept at $37^{\circ} \mathrm{C}$, ambient $\mathrm{O}_{2}$ and $5 \% \mathrm{CO}_{2}$. Cells were characterized by real-time RT-qPCR, RNA-seq, flow cytometry and immunofluorescence (see Supplementary material for details).

\section{Functional assessment of HLC}

Albumin production was evaluated using the Multigent ${ }^{\circledR}$ microalbumin assay on the Architect cSystems. Cyp3A4 activity and urea synthesis were measured using P450-Glo ${ }^{\mathrm{TM}}$ Assay (Promega) and Quantichrom urea assay kit (Gentaur), respectively, according to manufacturers' instructions.

\section{Mitochondrial respiration analysis}

Mitochondrial stress testing was carried out using a Seahorse Bioscience XF96 analyzer (Seahorse Bioscience) in 96 -well plates at $37^{\circ} \mathrm{C}$ as per the manufacturer's instructions, with 
minor modifications (see Supplementary material for details).

\section{Statistical analysis}

Replicates refer to independent experiments on $\geq 3$ different PSC populations. Values are shown as mean \pm standard error (SEM). Mann-Whitney $U$ test was used to compare RT-qPCR and functional data. A p-value $<0.05$ was considered significant.

\section{ACCESSION NUMBERS:}

The RNA sequencing data discussed in this publication have been deposited in the NCBI Gene Expression Omnibus under accession number GEO: GSE152390.

\section{ACKNOWLEDGEMENTS}

This work was supported by: Stem Cell Network grant FY17/DT8 (M.P.), Canadian Institute of Health Research New Investigator in Maternal, Reproductive, Child \& Youth Health grant MY6155373 (M.P.), "Fonds de Recherche du Québec - Santé” Junior 1 Clinician-Scientist grant (M.P.). We thank the Vision Health Research Network for funding the Single-cell Academy platform and Ines Boufaied (CHU Sainte-Justine's Flow Cytometry core facility) for her precious collaboration.

\section{AUTHOR CONTRIBUTIONS:}

Conception and design: C.R., M.P.; collection of data: C.R., MA.MC, Q.T.P., P.G., S.S., N.B., CL.M.; data analysis and interpretation: C.R., Q.T.P., G.C., JS.J., M.P.; manuscript writing: C.R., JS.J., M.P.; provision of study material: B.R.; financial support and supervision: M.P. All authors approved the final version of the manuscript.

\section{DECLARATION OF INTERESTS}


bioRxiv preprint doi: https://doi.org/10.1101/2020.09.02.280172; this version posted September 3, 2020. The copyright holder for this preprint

(which was not certified by peer review) is the author/funder, who has granted bioRxiv a license to display the preprint in perpetuity. It is made available under aCC-BY-NC-ND 4.0 International license.

C.R. and M.P. filed a patent application to protect this protocol and are co-founders, shareholders and directors of Morphocell Technologies Inc. The other authors have no competing interests to declare. 


\section{REFERENCES}

1 Paganelli M. Cell Therapy in Acute and Chronic Liver Disease. In: D’Antiga L (ed). Pediatric Hepatology and Liver Transplantation. Springer, 2019, pp. 781-797.

2 Si-Tayeb K, Lemaigre FP, Duncan SA. Organogenesis and Development of the Liver. Dev Cell 2010; 18:175-189.

3 Gordillo M, Evans T, Gouon-Evans V. Orchestrating liver development. Development 2015; 142:2094-2108

4 Ober EA, Lemaigre FP. Development of the liver: Insights into organ and tissue morphogenesis. J Hepatol 2018; 68:1049-1062

5 Takayama K, Akita N, Mimura N, Akahira R, Taniguchi Y, Ikeda M et al. Generation of safe and therapeutically effective human induced pluripotent stem cell-derived hepatocyte-like cells for regenerative medicine. Hepatology commun 2017; 1:1058-1069

6 Ogawa S, Surapisitchat J, Virtanen C, Ogawa M, Niapour M, Sugamori KS et al. Threedimensional culture and cAMP signaling promote the maturation of human pluripotent stem cellderived hepatocytes. Development 2013; 140:3285-3296

7 Touboul T, Chen S, To CC, Mora-Castilla S, Sabatini K, Tukey RH et al. Stage-specific regulation of the WNT/B-catenin pathway enhances differentiation of hESCs into hepatocytes. $J$ Hepatol 2016; 64:1315-1326

8 Baxter M, Withey S, Harrison S, Segeritz C-P, Zhang F, Atkinson-Dell R et al. Phenotypic and functional analyses show stem cell-derived hepatocyte-like cells better mimic fetal rather than adult hepatocytes. J Hepatol 2015; 62:581-589

9 Kajiwara M, Aoi T, Okita K, Takahashi R, Inoue H, Takayama N et al. Donor-dependent variations in hepatic differentiation from human-induced pluripotent stem cells. Proc Natl Acad Sci USA 2012; 109:12538-12543

10 McLean AB, D’Amour KA, Jones KL, Krishnamoorthy M, Kulik MJ, Reynolds DM et al. Activin A Efficiently Specifies Definitive Endoderm from Human Embryonic Stem Cells Only When Phosphatidylinositol 3-Kinase Signaling Is Suppressed. Stem cells 2007; 25: 29-38.

11 Sosa-Pineda B, Wigle JT, Oliver G. Hepatocyte migration during liver development requires Prox1. Nat Genet 2000; 25:254-255

12 Parviz F, Matullo C, Garrison WD, Savatski L, Adamson JW, Ning G et al. Hepatocyte nuclear factor 4alpha controls the development of a hepatic epithelium and liver morphogenesis. Nat Genet 2003; 34:292-296 
13 Han X, Wang Y, Pu W, Huang X, Qiu L, Li Y et al. Lineage Tracing Reveals the Bipotency of SOX9+ Hepatocytes during Liver Regeneration. Stem Cell Rep 2019; 12:624-638

14 Kamiya A, Gonzalez FJ. TNF-alpha regulates mouse fetal hepatic maturation induced by oncostatin M and extracellular matrices. Hepatology 2004; 40:527-536

15 Ramachandran A, Visschers RGJ, Duan L, Akakpo JY, Jaeschke H. Mitochondrial dysfunction as a mechanism of drug-induced hepatotoxicity: current understanding and future perspectives. J Clin Transl Res 2018; 4:75-100

16 Warren CR, O’Sullivan JF, Friesen M, Becker CE, Zhang X, Liu P et al. Induced Pluripotent Stem Cell Differentiation Enables Functional Validation of GWAS Variants in Metabolic Disease. Cell Stem Cell 2017; 20:547-557.e7

17 Gao Y, Zhang X, Zhang L, Cen J, Ni X, Liao X et al. Distinct Gene Expression and Epigenetic Signatures in Hepatocyte-like Cells Produced by Different Strategies from the Same Donor. Stem Cell Rep 2017; 9:1813-1824

18 Li Q, Hutchins AP, Chen Y, Li S, Shan Y, Liao B et al. A sequential EMT-MET mechanism drives the differentiation of human embryonic stem cells towards hepatocytes. Nat Commun 2017; 8:15166

19 Valizadeh A, Majidinia M, Samadi-Kafil H, Yousefi M, Yousefi B. The roles of signaling pathways in liver repair and regeneration. J Cell Physiol 2019; 234:14966-14974

20 Braver-Sewradj SP den, Braver MW den, Vermeulen NPE, Commandeur JNM, Richert L, Vos JC. Inter-donor variability of phase I/phase II metabolism of three reference drugs in cryopreserved primary human hepatocytes in suspension and monolayer. Toxicol in Vitro 2016; 33:71-79

21 Reed KR, Athineos D, Meniel VS, Wilkins JA, Ridgway RA, Burke ZD et al. B-catenin deficiency, but not Myc deletion, suppresses the immediate phenotypes of APC loss in the liver. Proc Natl Acad Sci USA 2008; 105:18919-18923

22 Patel SH, Camargo FD, Yimlamai D. Hippo Signaling in the Liver Regulates Organ Size, Cell Fate, and Carcinogenesis. Gastroenterology 2017;152: 533-545

23 Uhlén M, Fagerberg L, Hallström BM, Lindskog C, Oksvold P, Mardinoglu A et al. Tissuebased map of the human proteome. Science 2015; 347:1260419

24 Fu G-B, Huang W-J, Zeng M, Zhou X, Wu H-P, Liu C-C et al. Expansion and differentiation of human hepatocyte-derived liver progenitor-like cells and their use for the study of hepatotropic pathogens. Cell Res 2019; 29:8-22

25 Xie B, Sun D, Du Y, Jia J, Sun S, Xu J et al. A two-step lineage reprogramming strategy to generate functionally competent human hepatocytes from fibroblasts. Cell Res 2019; 29:696-710 
26 Kim Y, Kang K, Lee SB, Seo D, Yoon S, Kim SJ et al. Small molecule-mediated reprogramming of human hepatocytes into bipotent progenitor cells. J Hepatol 2019; 70:97-107

27 Dorrity MW, Saunders LM, Queitsch C, Fields S, Trapnell C. Dimensionality reduction by UMAP to visualize physical and genetic interactions. Nat Commun 2020; 11:1537 


\section{FIGURE LEGENDS}

Figure 1. Mimicking liver development. A) Schematic representation of human liver development showing the role that the main actors and signaling pathways play at each developmental stage; B) Description of our new differentiation protocol showing how the sequential supplementation of activators and inhibitors allows to reproduce the signals acting on the developing liver tissue in the embryo at corresponding stages.

Figure 2. A-E) PSC-derived endoderm (DE). A) Expression of EOMES, FOXA2 and SOX17 genes over the first 5 days of differentiation (RT-qPCR, expressed as $\log _{10}$ mean fold change $\pm S E M, n=9$, ** $p<0.01$, *** $\mathrm{p}<0.001, * * * * \mathrm{p}<0.0001)$. B) Expression of endoderm-enriched genes in PSC-derived cells after 5 days of differentiation compared to undifferentiated PSC (RT-qPCR, expressed as $\log _{10}$ mean fold change \pm SEM, $\mathrm{n}=3$ for PSC, $\mathrm{n}=6$ for $\left.\mathrm{DE},{ }^{*} \mathrm{p}<0.05, * * \mathrm{p}<0.01\right)$. C) Expression of DE markers at the end of day 5 of differentiation compared to undifferentiated PSC (immunofluorescence, representative images, DAPI nuclear staining in included images, scale bar $200 \mu \mathrm{M})$. D) Expression of DE markers is highly homogeneous in PSC-derived DE cells (flow cytometry, $n \geq 4$, mean \pm SEM). E) FOXA2/CXCR4/SOX17 triple-positive cells constitute $>90 \%$ of PSC-derived DE (flow cytometry, $n=4$; left: mean \pm SEM, center and right: representative experiment). F-K) Posterior foregut and liver bud formation. F-G) PSC-derived VPFG: expression of HHEX, PROX1, HNF4A, AFP and ALB genes in PSC-derived cells (RT-qPCR expressed as $\log _{10}$ mean fold change \pm SEM, compared to: $F$, undifferentiated PSC and G, PSC-derived DE; $n=3$ for PSC, $\mathrm{n} \geq 3$ for $\mathrm{DE}, \mathrm{n} \geq 5$ for VPFG; * $\mathrm{p}<0.05$, *** $\mathrm{p}<0.001$ ). H) PSC-derived cells after 15 days of differentiation achieve a gene expression profile consistent with hepatoblasts in the forming LB (RTqPCR, expressed as $\log _{10}$ mean fold change $\pm S E M ; n=3$ for VPFG, $n=8$ for $D E, * * p<0.01$ ). I) Expression of hepatoblast-enriched AFP, albumin, EpCAM and CK19 in PSC-derived LB cells (immunofluorescence, representative images with DAPI nuclear staining, scale bar $200 \mu \mathrm{M})$. J) LB cells show high homogeneity for EpCAM expression, while expression of a marker of pluripotency is negligible and comparable to background noise (flow cytometry; TRA-1-60: $n=6$ for PSC, $n=3$ for LB, mean \pm SEM; EpCAM: $n=5$, mean \pm SEM, representative experiment). K) The differentiation protocol allows the expansion of LB cells (automated cell count, $\mathrm{n}=6$ for PSC and LB, $\mathrm{n}=3$ for DE; mean \pm SEM, ** $\mathrm{p}<0.01$ ).

Figure 3. PSC-derived HLC. A) Representative morphology at the end of the differentiation protocol (phase contrast, scale bar $200 \mu \mathrm{M}$ left, $100 \mu \mathrm{M}$ right); B) Expression of liver-specific genes compared to 
LB cells (RT-qPCR, expressed as $\log _{10}$ mean fold change \pm SEM; $n=7,{ }^{*} p<0.05, * * * p<0.001$ ). C) Expression of liver-specific genes compared to adult PHH (RT-qPCR, expressed as $\log _{10}$ mean fold change \pm SEM; $n=9$ for $\mathrm{PHH}, \mathrm{n}=10$ for HLC; $\left.{ }^{* *} \mathrm{p}<0.01, * * * \mathrm{p}<0.001, \mathrm{~ns} \mathrm{p} \geq 0.05\right)$. D) Expression of liver-enriched AFP, albumin and CK19 in PSC-derived HLC (immunofluorescence; top: representative images with DAPI nuclear staining, scale bar $200 \mu \mathrm{M}$; bottom: percentage of positive cells compared to DAPI-positive nuclei, $n=9$, mean \pm SEM). E) Liver-specific functions performed by HLC: cyp3A4 activity (expressed as relative light units per million cells and compared to PHH, HepG2 cells and PSC; $n=6$ HLC, $n=7$ PHH, $n=5$ PSC, $n=3$ HepG2; mean \pm SEM, * $p<0.05)$; albumin secretion in HLC at day 25 and 30 compared to previous stages of differentiation and to PHH (ELISA, $n=4$ for HLC and PHH, $n=3$ for DE, VPFG and LB, mean \pm SEM); urea production (ELISA, $n=3$ for HLC, PSC and HepG2, $n=12$ for PHH, mean \pm SEM). F-G) Hepatotoxicity of acetaminophen (APAP) and amiodarone (AMIO) assessed through the measurement of oxygen consumption rate on HLC (O: oligomycin, F: FCCP, R/A: Rotenone/Antimycin A; $n=12$, mean \pm SEM).

Figure 4. The new differentiation protocol allows obtaining more mature HLC in greater number. A) Liver-specific genes are overexpressed in HLC obtained with our new protocol compared to a protocol not acting on Wnt and TGF $\beta$ signaling pathways beyond the DE stage (HLC-2; RT-qPCR, expressed as $\log _{10}$ mean fold change $\pm \mathrm{SEM}, \mathrm{n}=9$, * $\mathrm{p}<0.05, * * * \mathrm{p}<0.001, * * * * \mathrm{p}<0.0001$ ). B) Heatmap showing the expression of the top 46 liver-enriched genes in HLC obtained with our protocol compared to LB cells, HLC-2, HLC obtained with other previously described protocols, hepatocytes derived by transdifferentiation (hiHeps) and PHH (RNA-Seq, unsupervised clustering; list of genes from the Human Protein Atlas). ${ }^{16,17,23}$ C) Our HLC are more similar to PHH than most HLC obtained with previously described protocols: 2D representation of Uniform Manifold Approximation and Projection (UMAP)based dimensionality reduction of the top 17 principal components obtained analyzing the 3000 most variable genes across datasets. ${ }^{7,16-18,24-27}$ D) HLC obtained with our new protocol show significantly more Cyp3A4 activity than HLC-2 (relative light units per million cells; $n=6$ HLC, $n=4$ HLC-2; mean \pm SEM, ** $p<0.01$ ). E) Our HLC secrete more albumin per million cells than HLC-2 (ELISA, $n=4$ for HLC, $n=10$ for $\mathrm{HLC}-2$; mean $\pm \mathrm{SEM}, * * \mathrm{p}<0.01)$. F) The new differentiation protocol allows generating $\mathrm{HLC}$ with significantly more efficiency (automated cell count, $n=4$; mean $\pm S E M, * p<0.05$ ). 


\section{Figure 1}

A

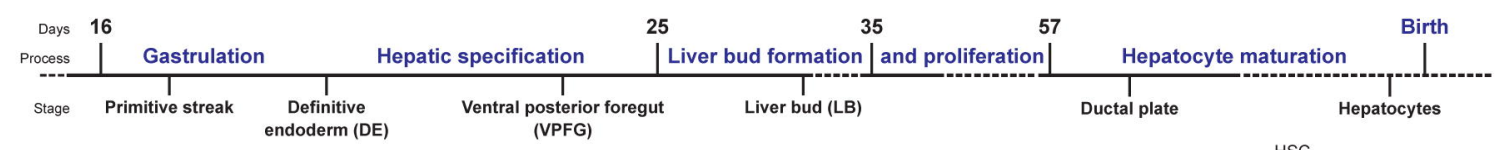

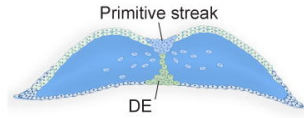

DE

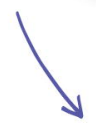

B

BMP

Septum transversum

mesenchyme (STM)
(VPFG)
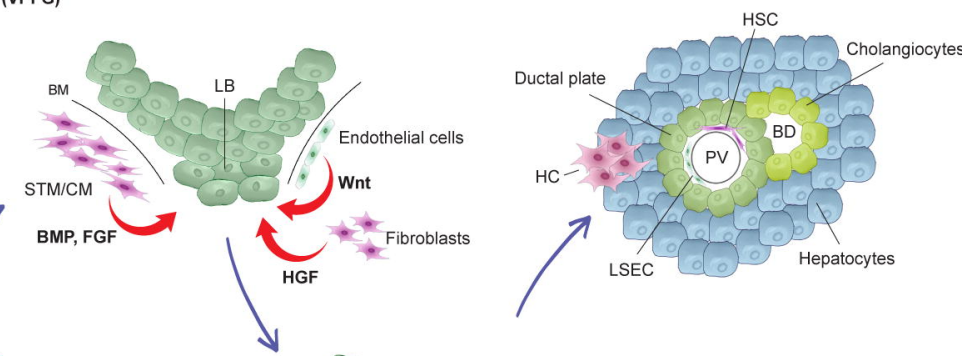

FGF

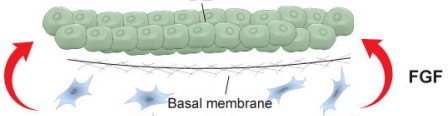

(BM) $-\mathrm{N}$

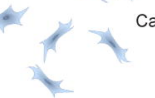

(CM)
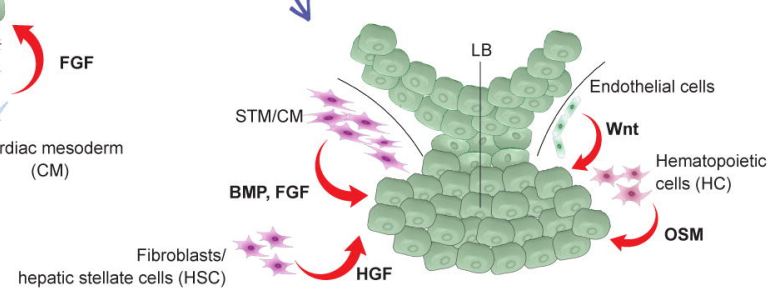

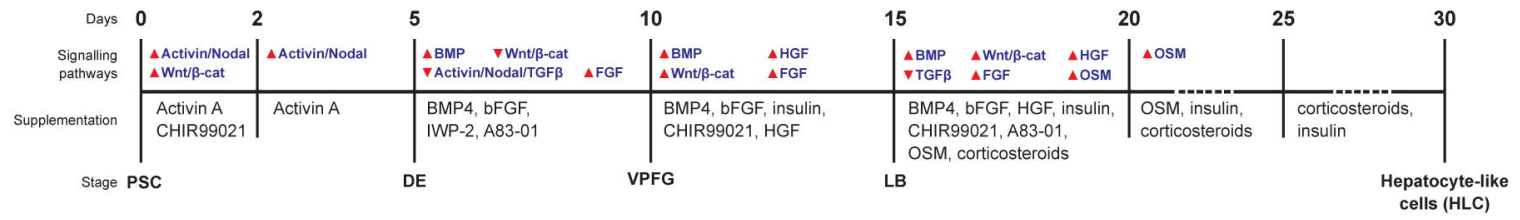


Figure 2

A

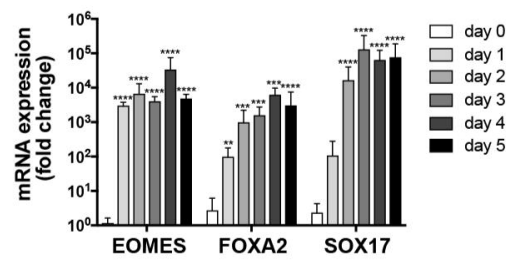

D

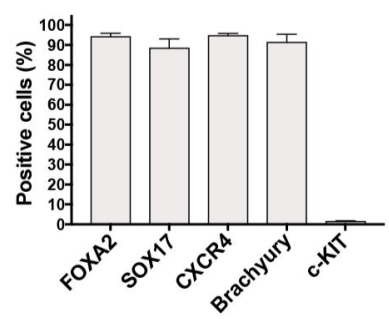

F

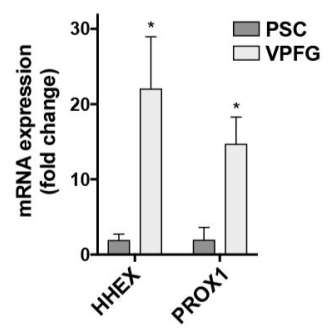

AFP

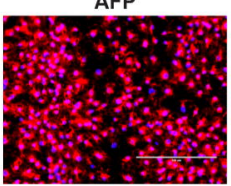

EpCAM

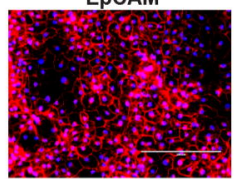

B

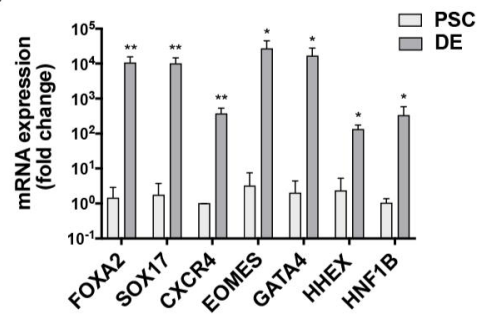

C

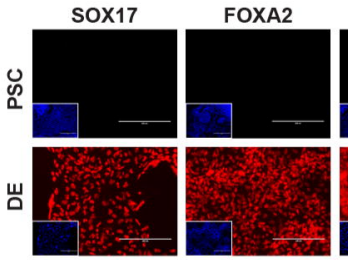

CXCR4
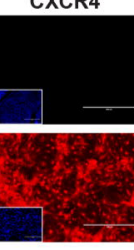

E

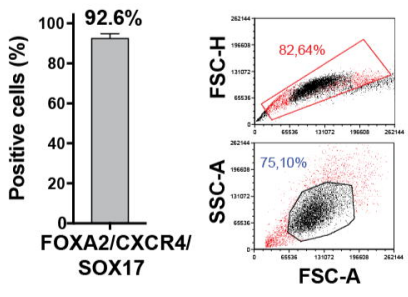

H

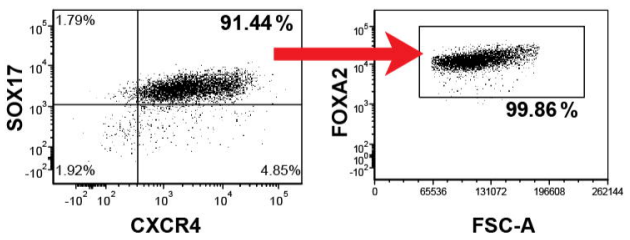

G
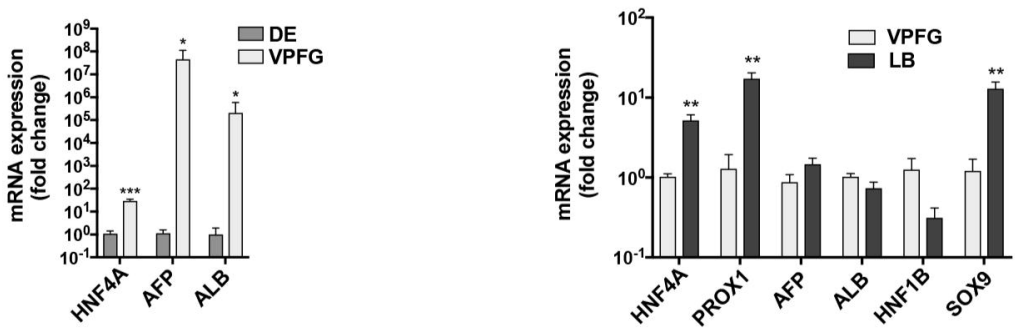

J

K
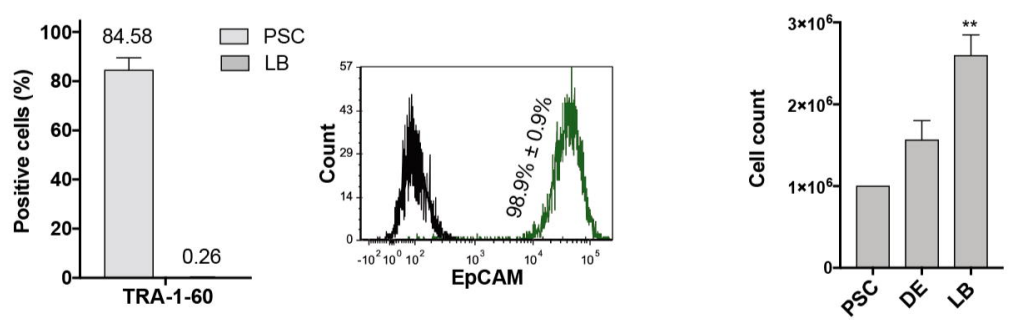
Figure 3

A

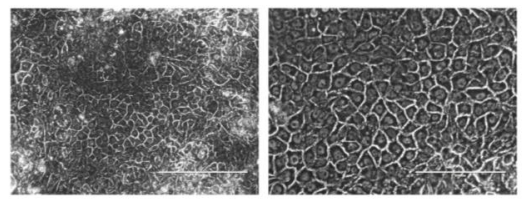

D
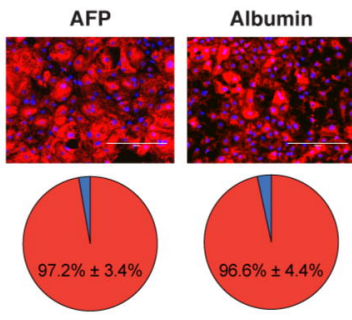

B

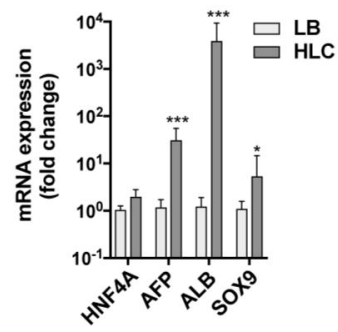

E

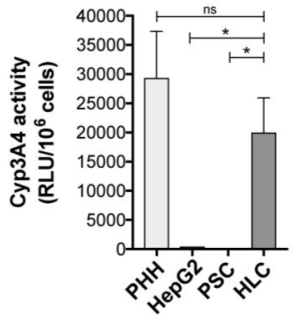

C
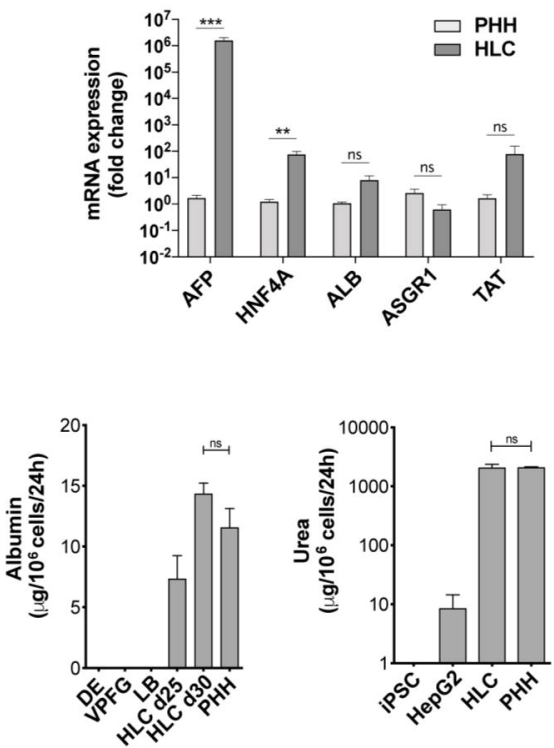

F

G
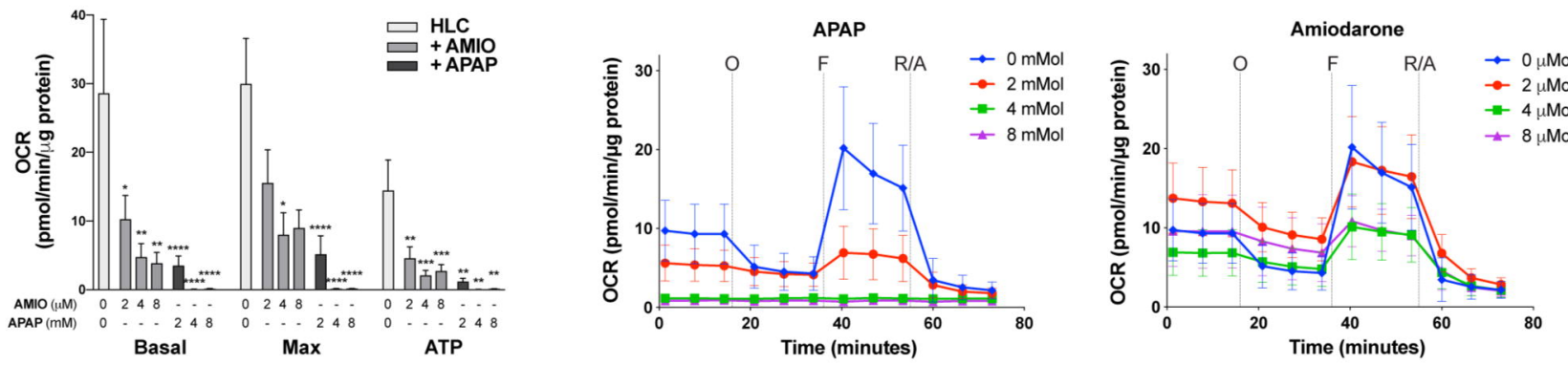
Figure 4

A

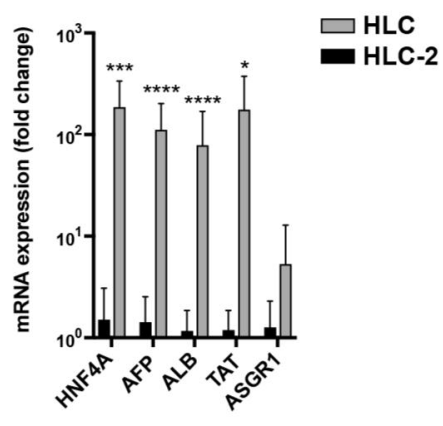

C

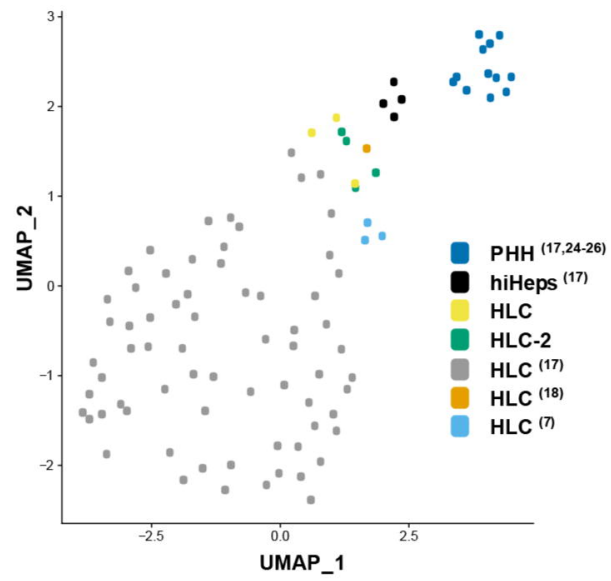

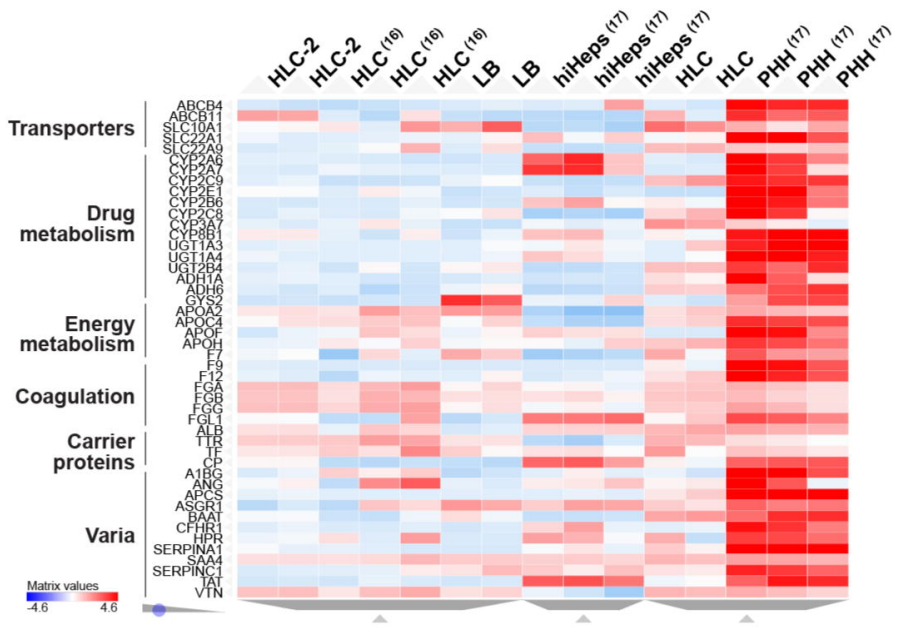

D

E

F
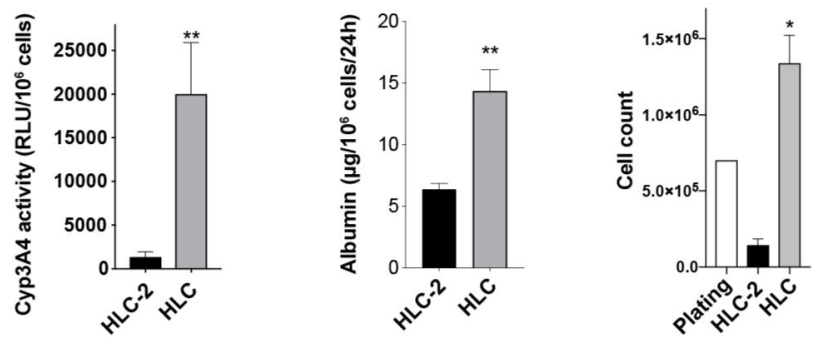